\title{
Moduli space of paired punctures, cyclohedra and particle pairs on a circle
}

\author{
Zhenjie Li and Chi Zhang \\ CAS Key Laboratory of Theoretical Physics, \\ Institute of Theoretical Physics, Chinese Academy of Sciences, \\ Beijing 100190, China \\ School of Physical Sciences, University of Chinese Academy of Sciences, \\ No. 19A Yuquan Road, Beijing 100049, China \\ E-mail: lizhenjie@itp.ac.cn, zhangchi@itp.ac.cn
}

ABSTRACT: In this paper, we study a new moduli space $\mathcal{M}_{n+1}^{\mathrm{c}}$, which is obtained from $\mathcal{M}_{0,2 n+2}$ by identifying pairs of punctures. We find that this space is tiled by $2^{n-1} n$ ! cyclohedra, and construct the canonical form for each chamber. We also find the corresponding Koba-Nielsen factor can be viewed as the potential of the system of $n+1$ pairs of particles on a circle, which is similar to the original case of $\mathcal{M}_{0, n}$ where the system is $n-3$ particles on a line. We investigate the intersection numbers of chambers equipped with Koba-Nielsen factors. Then we construct cyclohedra in kinematic space and show that the scattering equations serve as a map between the interior of worldsheet cyclohedron and kinematic cyclohedron. Finally, we briefly discuss string-like integrals over such moduli space.

Keywords: Scattering Amplitudes, Differential and Algebraic Geometry, Bosonic Strings ARXIV EPRINT: 1812.10727 


\section{Contents}

1 Introduction 1

2 Moduli space of pairs of punctures on the Riemann sphere 2

2.1 Real moduli space $\mathcal{M}_{n+1}^{\mathrm{c}}(\mathbb{R})$ and cyclohedron $W_{n} \quad 3$

2.2 Parke-Taylor forms as canonical forms of moduli space cyclohedra 6

2.3 Koba-Nielsen factor and scattering equations: particle pairs on a circle 8

$\begin{array}{lll}3 & \text { Intersection numbers } & 10\end{array}$

4 Kinematic cyclohedra and CHY formula $\quad 13$

$\begin{array}{lll}4.1 & \text { Kinematic cyclohedra } & 13\end{array}$

$\begin{array}{lll}4.2 & \text { Scattering equations as a map between cyclohedra } & 16\end{array}$

$5 \quad Z$-integrals on the moduli space $\mathcal{M}_{n+1}^{\mathrm{c}}(\mathbb{R}) \quad 18$

6 Outlook 20

\section{Introduction}

The scattering processes are closely linked to the Riemann surfaces with punctures since the birth of string theory. In particular, the scattering of $n$ massless particles can be described as a localized integral over some moduli spaces in the context of Witten-RSV formalism [1, 2] or CHY formalism [3-5]. In other words, the tree-level $S$-matrix of massless particles can be computed based on a map from some moduli space to kinematic space, which in general dimension is provided by the so-called scattering equations

$$
\sum_{j \neq i} \frac{k_{i} \cdot k_{j}}{z_{i}-z_{j}}=0 \quad \text { for } i \in\{1, \ldots, n\} .
$$

This map itself has been studied in detail in a new framework [6] and recast as a pushforward from differential forms on the moduli space $\mathcal{M}_{0, n}$, which are called worldsheet forms, to differential forms on the kinematic space of $n$ massless particles, which are called scattering forms. The combinatorial and geometrical aspects of moduli space and kinematic space become hence crucial in this context. Such ideas have been further developed by considering extended logarithmic differential forms as in [7] and introducing subspace in kinematic space as in $[7,8]$.

In another closely-related approach to amplitudes - intersection theory [9, 10], the combinatorial and geometrical properties of moduli space also play an important role. In this formalism, scattering amplitudes and related physical qualities are understood as intersection numbers of various twisted cycles and(or) cocycles which characterize the boundary 
information of the moduli space concerned. In the case of $\mathcal{M}_{0, n}$, a basis of twisted cocycles turn out to be the Parke-Taylor forms which are precisely the most important worldsheet forms considered in [6]. The combinatorial structure of some physical qualities, for example, the planar bi-adjoint $\phi^{3}$ amplitudes in $[5,6]$ and the inverse of Kawai-Lewellen-Tye(KLT) matrix $m_{\alpha^{\prime}}(\alpha \mid \beta)$ in $[9,11]$, are governed by the boundary structure of $\mathcal{M}_{0, n}(\mathbb{R})$, which turn out to be the so-called assocaihedra [12, 13].

In this article, we will use these two tools - positive geometry [14] and intersection theory - to study another interesting moduli space, which is obtained from $\mathcal{M}_{0,2 n+2}$ by imposing identifications for pairs of punctures, such as $z_{i}=-z_{i+n+1}$, equipped with the corresponding Koba-Nielsen factor. This is a $n$-dimensional space and denoted as $\mathcal{M}_{n+1}^{\mathrm{c}}$ in the remainder of this article, and the corresponding Koba-Nielsen factor can be inherited from the usual Koba-Nielsen factor for $2 n+2$ particles by imposing the above identifications. In particular, the real moduli space $\mathcal{M}_{n+1}^{c}(\mathbb{R})$ is tiled by $n$-dimensional cyclohedra $W_{n}$. The main result of this article is to recover this structure in kinematic space via intersection theory and positive geometry. To this end, we first introduce the ingredients in section 2, i.e., Parke-Taylor forms and scattering equations, and study their properties further. To do that, We will give the precise definition of $\mathcal{M}_{n+1}^{\mathrm{c}}(\mathbb{R})$ and $\mathcal{M}_{n+1}^{\mathrm{c}+}(\mathbb{R})$ and show: (i) $\mathcal{M}_{n+1}^{\mathrm{c}}(\mathbb{R})$ is tiled by $2^{n-1} n ! \mathcal{M}_{n+1}^{\mathrm{c}+}(\mathbb{R})$ which can be identified with a cyclohedron $W_{n}$, (ii) the corresponding Parke-Taylor form is the canonical form on $\mathcal{M}_{n+1}^{\mathrm{c}+}(\mathbb{R})$, and (iii) the scattering equations as the stationary points of a particle system have $2^{n-1} n$ ! solutions.

With this knowledge of moduli space $\mathcal{M}_{n+1}^{\mathrm{c}}$, we move to the kinematic space. The way through intersection theory is quite straightforward, the intersection number of two twisted cocycles $\alpha$ and $\beta$ tell us the result and the structure of $m_{\alpha^{\prime}}(\alpha \mid \beta)$, and shown in section 3 . While in section 4 , we will first construct a cyclohedron in the kinematic space, then rewrite the scattering equations as a map from the moduli cyclohedron to the kinematic cyclohedron, as done in [6]. As a result of this pushforward, the amplitudes $m(\alpha \mid \beta)$ can be extracted from the scattering form by pullbacking to the corresponding subspace. Then it becomes obvious that $m(\alpha \mid \beta)$ is given by $m_{\alpha^{\prime}}(\alpha \mid \beta)$ in the limit of $\alpha^{\prime} \rightarrow 0$.

Last but not the least, we will consider the natural integrals on such moduli space that are analogs of the so-called $Z$-integrals for open-string case [15]. Interestingly, the Koba-Nielsen factor for $n+1$ open strings and one closed-string is a special limit of our case. However, this limit needs to be taken carefully since some integrals don't behave well under this limit. Here we just give a brief survey on its $\alpha^{\prime}$-expansion and number theoretical properties before taking this limit.

\section{Moduli space of pairs of punctures on the Riemann sphere}

In this section, we consider the moduli space of distinct pairs of punctures $\left(z, z^{\prime}\right)$ on the Riemann sphere $\mathbb{C P}^{1}$, where two punctures in each pair are related by $z^{\prime}=-z$.

It's clear that an arbitrary $\mathrm{SL}(2, \mathbb{C})$ transformation on the Riemann sphere will not keep two punctures $z$ and $z^{\prime}$ in a pair still satisfying the relation that $z^{\prime}=-z$. One can 
check that the surviving transformations are

$$
z \mapsto \lambda z \quad \text { and } \quad z \mapsto \frac{1}{z}
$$

where $\lambda$ is a non-zero complex number. Therefore, the remaining $\operatorname{SL}(2, \mathbb{C})$ effectively is the group $(\mathbb{C}-\{0\}) \rtimes \mathbb{Z}_{2}$ whose elements are transformations

$$
(\lambda, a): z \mapsto \lambda z^{a}
$$

where $\lambda \in(\mathbb{C}-\{0\})$ and $a= \pm 1 .^{1}$

Now, suppose there are $n+1$ distinct such pairs of two punctures $\left\{\left(z_{i}, z_{i}^{\prime}\right): 0 \leq i \leq n\right\}$ on $\mathbb{C P}^{1}$. Our complex moduli space is the quotient of this configurations space, modulo the remaining redundancy $(\mathbb{C}-\{0\}) \rtimes \mathbb{Z}_{2}$. Let's denote it by $\mathcal{M}_{n+1}^{\mathrm{c}}(\mathbb{C})$. For future convenience, it's useful to enlarge the index set to $\mathbb{Z}_{2 n+2}=\{0, \ldots, n, n+1, \ldots, 2 n+1\}$ and identify $z_{i}^{\prime}$ with $z_{i+n+1}$ or $z_{\tilde{\imath}}$, where $\tilde{\imath}=i+n+1 \in \mathbb{Z}_{2 n+2}$.

\subsection{Real moduli space $\mathcal{M}_{n+1}^{\mathrm{c}}(\mathbb{R})$ and cyclohedron $W_{n}$}

In the context of string theory, the moduli space $\mathcal{M}_{0, n}(\mathbb{C})$ of $n$ distinct punctures on the Riemann sphere $\mathbb{C P}^{1}$ and its real part $\mathcal{M}_{0, n}(\mathbb{R})$ of $n$ distinct punctures on the circle (i.e. the boundary of the disk) $\mathbb{R} \mathbb{P}^{1}=S^{1}$ are used to calculate tree-level amplitudes of closed strings and open strings respectively. Similarly, we can consider the real part of our complex moduli space $\mathcal{M}_{n+1}^{\mathrm{c}}(\mathbb{C})$ by setting all these pairs of punctures on the unit circle of $\mathbb{C}$. We denote this new real moduli space by $\mathcal{M}_{n+1}^{\mathrm{c}}(\mathbb{R})$. As in the complex case, the $\operatorname{SL}(2, \mathbb{R})$ redundancy now reduces to $\mathrm{U}(1) \rtimes \mathbb{Z}_{2}$, which is generated by

$$
z \mapsto \exp (\mathrm{i} \theta) z \quad \text { and } \quad z \mapsto 1 / z=\bar{z},
$$

where $\theta \in \mathbb{R}$ and $|z|=1$. Since all punctures now live on the unit circle, it's very convenient to use their arguments $\theta_{i}$ to coordinate them, i.e., $z_{i}=\exp \left(\mathrm{i} \theta_{i}\right)$. Besides, it's also convenient to introduce another set of useful real variables

$$
x_{i}=\tan \left(\theta_{i} / 2\right)=\mathrm{i} \frac{1-z_{i}}{1+z_{i}} .
$$

In fact, these are the natural coordinates of another possible great circle $\mathbb{R} \cup\{\infty\}$ on the Riemann sphere where pairs of punctures can live.

A lot of works on $\mathcal{M}_{0, n}(\mathbb{R})$ have revealed some hidden structures of this moduli space. For example, its Fulton-MacPherson compactification can be tiled by associahedra [16]. In the rest of this subsection, we will show some similar structures on $\mathcal{M}_{n+1}^{\mathrm{c}}(\mathbb{R})$.

Firstly, we fix the $\mathrm{U}(1) \rtimes \mathbb{Z}_{2}$ redundancy by setting $z_{0}=-z_{\tilde{0}}=1$ and limiting $z_{1}$ on the upper semi-circle $S_{+}^{1}$. Since either $z$ or $-z$ is on the upper semi-circle for any pairs of punctures $(z,-z) \neq(1,-1)$ on the unit circle, one can use the one living on the $S_{+}^{1}$ to represent this pair. Therefore, after the gauge fixing, $\mathcal{M}_{n+1}^{\mathrm{c}}(\mathbb{R})$ is described by

$$
\mathcal{M}_{n+1}^{\mathrm{c}}(\mathbb{R})=\left\{\left(z_{1}, \pm z_{2}, \ldots, \pm z_{n}\right) \in\left(S_{+}^{1}\right)^{n}\right\}-\Delta,
$$

\footnotetext{
${ }^{1}$ It's a simi-product because the multiplication rule is $\left(\lambda_{1}, a_{1}\right) \cdot\left(\lambda_{2}, a_{2}\right)=\left(\lambda_{1} \lambda_{2}^{a_{1}}, a_{1} a_{2}\right)$.
} 
where

$$
\Delta=\left\{\left(z_{1}, \pm z_{2}, \ldots, \pm z_{n}\right) \in\left(S_{+}^{1}\right)^{n}: \exists i, j \text { s.t. } z_{i}^{2}=z_{j}^{2} \text { or } z_{i}^{2}=1\right\}
$$

One should exclude $\Delta$ because points in this subset represent some pairs of punctures on the unit circle pinched.

Since there are two choices $z_{i}$ and $-z_{i}$ for all $2 \leq i \leq n, \mathcal{M}_{n+1}^{\mathrm{c}}(\mathbb{R})$ is $2^{n-1}$ copies of the following space

$$
\left\{\left(p_{1}, p_{2}, \ldots, p_{n}\right) \in\left(S_{+}^{1}\right)^{n}\right\}-\Delta=\left\{\left(1, p_{1}^{2}, p_{2}^{2}, \ldots, p_{n}^{2}\right) \in\left(S^{1}\right)^{n+1}\right\}-\Delta^{\prime},
$$

where

$$
\Delta^{\prime}=\left\{\left(1, q_{1}, q_{2}, \ldots, q_{n}\right) \in\left(S^{1}\right)^{n+1}: \exists i, j \text { s.t. } q_{i}=q_{j} \text { or } q_{i}=1\right\}
$$

This is a known moduli space $\mathrm{F}\left(S^{1}, n+1\right) / S^{1}$ which has been well investigated in [17]. Therefore, our real moduli space $\mathcal{M}_{n+1}^{\mathrm{c}}(\mathbb{R})$ is $2^{n-1} \mathrm{~F}\left(S^{1}, n+1\right) / S^{1}$.

The real Fulton-MacPherson compactification of $\mathrm{F}\left(S^{1}, n+1\right) / S^{1}$, denoted by $\overline{\mathcal{Z}}^{n+1}$, can be tiled by $n$ ! cyclohedra [17], so the same compactification of our moduli space can be tiled by $2^{n-1} n$ ! cyclohedra. Suppose proj: $\overline{\mathcal{Z}}^{n+1} \rightarrow \mathrm{F}\left(S^{1}, n+1\right) / S^{1}$ is the compactification map, then every cyclohedron is given by

$$
W_{n}(\sigma)=\overline{\operatorname{proj}^{-1}\left(\left\{0<\varphi_{\sigma(1)}<\varphi_{\sigma(2)}<\cdots<\varphi_{\sigma(n)}<2 \pi\right\}\right)},
$$

where $\varphi_{i}$ is the argument of $p_{i}^{2}$ and $\sigma \in S_{n}$ is a permutation of index set $\{1, \ldots, n\}$. In our case, every cyclohedron is given by

$$
\begin{aligned}
W_{n}\left(\sigma,\left\{\mathbf{s}_{i}\right\}\right) & =\overline{\operatorname{proj}^{-1}\left(\left\{0<\theta_{\sigma(1)}^{\mathbf{s}_{\sigma(1)}}<\theta_{\sigma(2)}^{\mathbf{s}_{\sigma(2)}}<\cdots<\theta_{\sigma(n)}^{\mathbf{s}_{\sigma(n)}}<\pi\right\}\right)}, \\
& =: \overline{\operatorname{proj}^{-1}\left(\mathcal{M}_{n+1}^{\mathrm{c}+}\left(\sigma,\left\{\mathbf{s}_{i}\right\}\right)\right)}
\end{aligned}
$$

where $\mathrm{s}_{i}= \pm 1$ and

$$
\theta_{i}^{+}=\theta_{i}, \quad \theta_{i}^{-}=\theta_{i}-\pi
$$

From this construction, we can conclude that facets of the cyclohedron $\mathcal{M}_{n+1}^{\mathrm{c}+}\left(\sigma,\left\{\mathrm{s}_{i}\right\}\right)$ (before compactification) lay on hyperplanes defined by equations $\mathrm{s}_{\sigma(i)} z_{\sigma(i)}-\mathrm{s}_{\sigma(i+1)} z_{\sigma(i+1)}=$ 0 . In terms of $\left\{\theta_{i}\right\}$, these hyperplane equations are

$$
0 \leq \theta_{i}<2 \pi \quad \text { and } \quad \theta_{i}-\theta_{j}= \begin{cases}0, & \text { if } \mathrm{s}_{i}-\mathrm{s}_{j}=0 \bmod 2 \\ \pm \pi, & \text { if } \mathrm{s}_{i}-\mathrm{s}_{j}= \pm 1\end{cases}
$$


For example, hyperplanes for $n=2$ are shown in the following diagram:

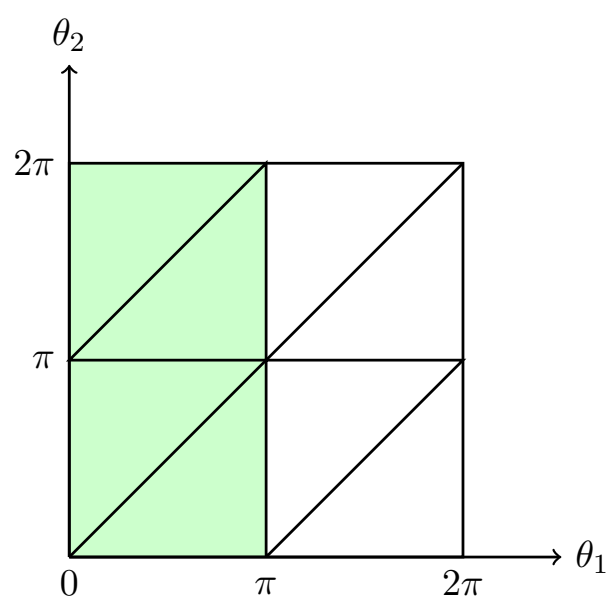

with the identification $\left(\theta_{1}, \theta_{2}\right) \sim\left(2 \pi-\theta_{1}, 2 \pi-\theta_{2}\right)$ due to the $\mathbb{Z}_{2}$ redundancy.

As before, we can fix this redundancy by setting $\theta_{1}<\pi$, so there are $4=2^{1} \times 2$ ! chambers (green ones in the above diagram) corresponding to the cyclohedra before compactification. After compactification, each chamber becomes a hexagon, the 2-dimensional cyclohedron. For example, the triangle $0<\theta_{1}<\theta_{2}<\pi$ actually becomes

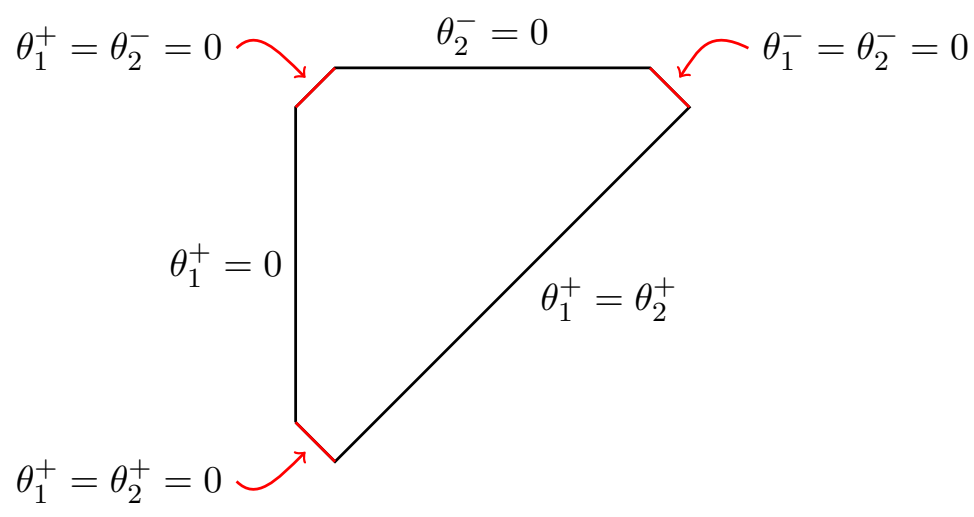

where three red facets are the result of compactification. Note that, every vertex should be blowed-up in the compactification so that no two cyclohedra intersect at a point.

For a general $n$, we can construct $W_{n}\left(\sigma,\left\{\mathbf{s}_{i}\right\}\right)$ from the corresponding $\mathcal{M}_{n+1}^{\mathrm{c}+}\left(\sigma,\left\{\mathbf{s}_{i}\right\}\right)$ and the compactification of the whole space $\mathcal{M}_{n+1}^{\mathrm{c}}(\mathbb{R})$ by the following steps:

(1) For every chamber before compactification, label $(i j)$ on each codimension-one face (facet) $H$ defined by $z_{i}=z_{j}$.

(2) For the face $H$ with higher codimension $k$ defined by $z_{i_{1}}=\cdots=z_{i_{k}}$ of these chambers, truncate it and label $\left(i_{1} i_{2} \cdots i_{k}\right)$ on the new created facet. Repeat this operation from low-dimensional faces to high-dimensional faces.

(3) Glue the faces with the same label in different cyclohedra, they will be the same face after compactification. 
Here we give a direct combinatorial definition of the cyclohedron. For any polytope $P$, there's a natural partial order of faces on it: for face $a$ and $b$,

$$
a \leq b \text { if and only if } a \subset b \text {, }
$$

then $P$ defines a partially ordered set (or poset for short) $(P, \leq)$. Conversely, we can use a poset to define a polytope whose associated poset of faces is isomorphic to the given poset.

Definition 1 (Cyclohedron). Let $\mathrm{Cyc}(n)$ be the poset of all centrally symmetric dissections of a convex $(2 n+2)$-gon using non-intersecting diagonals, ordered such that $b \leq b^{\prime}$ if $b$ is obtained from $b^{\prime}$ by adding (non-intersecting) diagonals. The $n$-dimensional cyclohedron $W_{n}$ is convex polygon whose face poset is isomorphic to $\operatorname{Cyc}(n)$.

For example, $W_{2}$ is a hexagon.

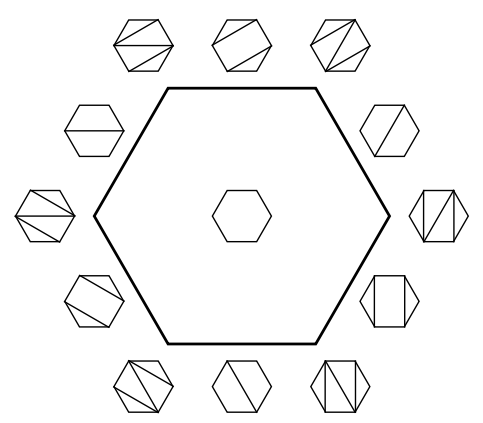

The definitive property of a cyclohedron is its geometric factorization [17] that every facet of a $n$-dimensional cyclohedron $W_{n}$ is the product of a $(n-i)$-dimensional cyclohedron $W_{n-i}$ and a $(i-1)$-dimensional associahedron $A_{i-1}$, i.e.

$$
H \cong W_{n-i} \times A_{i-1},
$$

where $H$ is a facet of $W_{n}$ and $i$ is a positive integer. In the following sections, we will see it again and again in different forms.

\subsection{Parke-Taylor forms as canonical forms of moduli space cyclohedra}

As shown in the previous subsection, the interior of a cyclohedron $W_{n}\left(\sigma,\left\{\mathbf{s}_{i}\right\}\right)$ is a simplex whose facets lay on the hyperplanes defined by equations

$$
\mathrm{s}_{\sigma(i)} z_{\sigma(i)}-\mathrm{s}_{\sigma(i+1)} z_{\sigma(i+1)}=0
$$

Therefore, we can write down the Parke-Taylor form as the canonical form [14] of this simplex

$$
\mathrm{PT}_{n}\left(\sigma(1)^{\mathrm{s}_{\sigma(1)}}, \ldots, \sigma(n)^{\mathbf{s}_{\sigma(n)}}\right):=\frac{2 z_{0} \mathrm{~d}\left(\mathrm{~s}_{1} z_{1}\right) \wedge \cdots \wedge \mathrm{d}\left(\mathrm{s}_{n} z_{n}\right)}{\left(z_{0}-\mathrm{s}_{\sigma(1)} z_{\sigma(1)}\right)\left(\mathrm{s}_{\sigma(1)} z_{\sigma(1)}-\mathrm{s}_{\sigma(2)} z_{\sigma(2)}\right) \cdots\left(\mathrm{s}_{\sigma(n)} z_{\sigma(n)}+z_{0}\right)} .
$$


The appearance of $z_{0}$ on the numerator makes $\mathrm{PT}_{n}$ invariant under $(\lambda, a): z \mapsto \lambda z^{a}$ for all $(\lambda, a) \in(\mathbb{C}-\{0\}) \rtimes \mathbb{Z}_{2}$. As shown in [9], Parke-Taylor forms can be rewritten as

$$
\begin{aligned}
& \mathrm{PT}_{n}\left(\sigma(1)^{\mathrm{s}_{\sigma(1)}}, \ldots, \sigma(n)^{\mathrm{s}_{\sigma(n)}}\right) \\
& \quad=(-1)^{n} \operatorname{sgn}(\sigma) \mathrm{d} \log \left(\frac{z_{0}-\mathrm{s}_{\sigma(1)} z_{\sigma(1)}}{\mathrm{s}_{\sigma(1)} z_{\sigma(1)}-\mathrm{s}_{\sigma(2)} z_{\sigma(2)}}\right) \wedge \cdots \wedge \mathrm{d} \log \left(\frac{\mathrm{s}_{\sigma(n-1)} z_{\sigma(n-1)}-\mathrm{s}_{\sigma(n)} z_{\sigma(n)}}{\mathrm{s}_{\sigma(n)} z_{\sigma(n)}+z_{0}}\right),
\end{aligned}
$$

so they are $\mathrm{d} \log$ forms.

What's more, Parke-Taylor forms still work well after compactification (locally it's a series of blowing-ups), so they are also the canonical forms of cyclohedra. ${ }^{2}$ Since every facet of a cyclohedron can be factorized into a cyclohedron and an associahedron, the residue of the canonical form at each facet should also be factorized into the corresponding canonical forms.

To see it, consider the facet of $W_{n}(\mathrm{id},\{+\})$ labeled by $(i \cdots j)$, which corresponds to the blowing-up of the pinching of the coordinates $\left\{z_{i}, \ldots, z_{j}\right\}$ of the moduli space. Let $z_{k}=z_{i}+t y_{k}$ for $i+1 \leq k \leq j$, where $\left[y_{i+1}, \ldots, y_{j}\right]$ are projective coordinates of $\mathbb{P}^{j-i-1}$, then the residue of $\mathrm{PT}_{n}\left(1^{+} 2^{+} \ldots n^{+}\right)$at $t=0$ can be written as

$$
\begin{aligned}
\operatorname{Res}_{t=0} \mathrm{PT}_{n}\left(1^{+} 2^{+} \cdots n^{+}\right) & =\operatorname{Res}_{t=0}\left[(-1)^{n} \mathrm{~d} \log \left(\frac{z_{0}-z_{1}}{z_{1}-z_{2}}\right) \wedge \cdots \wedge \mathrm{d} \log \left(\frac{z_{n-1}-z_{n}}{z_{n}+z_{0}}\right)\right] \\
& =( \pm) \mathrm{PT}_{n-(j-i)}\left(1^{+} 2^{+} \cdots i^{+}(j+1)^{+} \cdots n^{+}\right) \wedge \Omega
\end{aligned}
$$

here $\mathrm{PT}_{n-(j-i)}$ is the canonical form of a cyclohedron, while

$$
\Omega=\mathrm{d} \log \left(\frac{0-y_{i+1}}{y_{i+1}-y_{i+2}}\right) \wedge \cdots \wedge \mathrm{d} \log \left(\frac{y_{j-2}-y_{j-1}}{y_{j-1}-y_{j}}\right)
$$

is the canonical form of an associahedron up to a sign since it can be rewritten as

$$
\Omega=\frac{( \pm) \mathrm{d} y_{i+1} \wedge \cdots \wedge \mathrm{d} y_{j-1}}{\left(0-y_{i+1}\right)\left(y_{i+1}-y_{i+2}\right) \cdots\left(y_{j-1}-1\right)}
$$

in the piece of $\mathbb{P}^{j-i-1}$ where $y_{j}=1$.

Apparently, there are $2^{n} n$ ! different Parke-Taylor forms corresponding to different cyclohedra. Like the case of associahedra, there are some linear relations between these Parke-Taylor forms. A simple relation is

$$
\mathrm{PT}_{n}\left(\sigma(1)^{\mathbf{s}_{\sigma(1)}}, \ldots, \sigma(n)^{\mathbf{s}_{\sigma(n)}}\right)=(-1)^{n} \mathrm{PT}_{n}\left(\sigma(n)^{-\mathbf{s}_{\sigma(n)}}, \ldots, \sigma(1)^{-\mathbf{s}_{\sigma(1)}}\right),
$$

some not so trivial relations are

$$
\begin{aligned}
& \mathrm{PT}_{n}\left(1^{+} 2^{\mathrm{s}_{2}} \ldots n^{\mathrm{s}_{n}}\right)+\mathrm{PT}_{n}\left(2^{\mathrm{s}_{2}} 1^{+} \ldots n^{\mathrm{s}_{n}}\right)+\cdots+\mathrm{PT}_{n}\left(2^{\mathrm{s}_{2}} \ldots n^{\mathrm{s}_{n}} 1^{+}\right)+ \\
& \mathrm{PT}_{n}\left(1^{-} 2^{\mathrm{s}_{2}} \ldots n^{\mathrm{s}_{n}}\right)+\mathrm{PT}_{n}\left(2^{\mathrm{s}_{2}} 1^{-} \ldots n^{\mathrm{s}_{n}}\right)+\cdots+\mathrm{PT}_{n}\left(2^{\mathrm{s}_{2}} \ldots n^{\mathrm{s}_{n}} 1^{-}\right)=0
\end{aligned}
$$

and its permutations. The second kind of relations are analogues of Kleiss-Kuijf relations in the case of associahedra. We conjecture that the rank of these Parke-Taylor forms is $(2 n-1)$ !! because of these relations.

\footnotetext{
${ }^{2}$ More precisely, it is the pull-back of Parke-Taylor forms according to the compactification map that are the canonical forms of cyclohedra.
} 
To end this subsection, we express Parke-Taylor forms in terms of $\left\{x_{i}\right\}$ and $\left\{\theta_{i}\right\}$. They live on the real line, so it's more convenient for some purpose or other. In terms of $\left\{x_{i}\right\}$, Parke-Taylor forms are written as

$$
\operatorname{PT}\left(\sigma(1)^{\mathbf{s}_{\sigma(1)}}, \ldots, \sigma(n)^{\mathbf{s}_{\sigma(n)}}\right)=\frac{\left(x_{0}+x_{0}^{-1}\right) \mathrm{d}\left(\mathrm{s}_{1} x_{1}^{\mathbf{s}_{1}}\right) \wedge \cdots \wedge \mathrm{d}\left(\mathbf{s}_{n} x_{n}^{\mathbf{s}_{n}}\right)}{\left(x_{0}-\mathbf{s}_{\sigma(1)} x_{\sigma(1)}^{\mathbf{s}_{\sigma(1)}}\right) \cdots\left(\mathbf{s}_{\sigma(n)} x_{\sigma(n)}^{\mathbf{s}_{\sigma(n)}}+x_{0}^{-1}\right)},
$$

where $s_{i}= \pm 1$. Then it's direct to carry out Parke-Taylor forms in terms of $\left\{\theta_{i}^{s_{i}}\right\}$ by using $\mathrm{s}_{i} x_{i}^{\mathrm{s}_{i}}=\tan \left(\theta_{i}^{\mathrm{s}_{i}} / 2\right)$,

$$
\operatorname{PT}\left(\sigma(1)^{\mathbf{s}_{\sigma(1)}}, \ldots, \sigma(n)^{\mathbf{s} \sigma(n)}\right)=\frac{\sin \left(\theta_{0, n+1}^{\mathbf{s}} / 2\right) \mathrm{d} \theta_{1} \wedge \cdots \wedge \mathrm{d} \theta_{n}}{\sin \left(\theta_{0, \sigma(1)}^{\mathbf{s}} / 2\right) \cdots \sin \left(\theta_{\sigma(n-1), \sigma(n)}^{\mathbf{s}} / 2\right) \sin \left(\theta_{\sigma(n), n+1}^{\mathbf{s}} / 2\right)},
$$

where $\theta_{i, j}^{\mathbf{s}}:=\theta_{i}^{\mathbf{s}_{i}}-\theta_{j}^{\mathbf{s}_{j}}$.

\subsection{Koba-Nielsen factor and scattering equations: particle pairs on a circle}

In order to connect to the kinematic space, we should introduce 'Mandelstam variables' $s_{i j}$ for any pair of punctures with the usual properties, i.e., $s_{i i}=0, s_{i j}=s_{j i}$ and the momentum conservation

$$
\sum_{j=0}^{2 n+1} s_{i j}=0
$$

but with additional identifications $s_{\tilde{\imath} \tilde{\jmath}}=s_{i j}$ since $z_{i}-z_{j}=0$ and $z_{\tilde{\imath}}-z_{\tilde{\jmath}}=0$ are the same hyperplane in the moduli space. It's natural to write down a factor

$$
\mathcal{I}=\prod_{0 \leq i<j \leq 2 n+1}\left(z_{i}-z_{j}\right)^{\alpha^{\prime} s_{i j}}
$$

as the analogue of the so-called Koba-Nielsen factor in string theory. However, with the new identifications $s_{i j}=s_{\tilde{\imath} \tilde{\jmath}}$ of Mandelstam variables, this factor $\mathcal{I}$ can be rewritten to drop this redundancy. For future use, it's convenient to rewrite it as

$$
\mathcal{I}=\prod_{0 \leq i<j \leq n} \sin \left(\frac{\theta_{i}-\theta_{j}}{2}\right)^{2 \alpha^{\prime} s_{i j}} \cos \left(\frac{\theta_{i}-\theta_{j}}{2}\right)^{2 \alpha^{\prime} s_{i \tilde{\jmath}}}
$$

in terms of $\left\{\theta_{i}\right\}$, or,

$$
\mathcal{I}=\prod_{0 \leq i<j \leq n}\left(\frac{\left(x_{i}-x_{j}\right)^{2}}{\left(1+x_{i}^{2}\right)\left(1+x_{j}^{2}\right)}\right)^{s_{i j}}\left(\frac{\left(1+x_{i} x_{j}\right)^{2}}{\left(1+x_{i}^{2}\right)\left(1+x_{j}^{2}\right)}\right)^{s_{i \tilde{\jmath}}}
$$

in terms of $\left\{x_{i}\right\}$.

As shown in [18], the number of stationary points of the Koba-Nielsen factor equals to the number of associahedra in the moduli space $\mathcal{M}_{0, n}(\mathbb{R})$, which is a highly non-trivial result because of the Poincaré dual. Therefore, it's believed that the number of stationary points of our new Koba-Nielsen factor eq. (2.7) should equal the number of cyclohedra $2^{n-1} n$ !. In the rest of this subsection, we will prove that it's indeed the number of stationary points. 


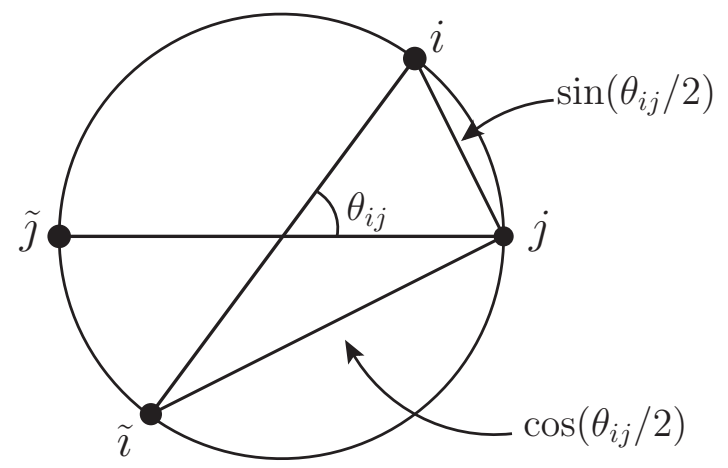

Figure 1. Particle pairs on a circle.

The stationary points of Koba-Nielsen factor are determined by the equation $\mathrm{d} \log (\mathcal{I})=0$, then it gives a set of equations in $z$-variables, which are called scattering equations,

$$
E_{i}:=\frac{\partial}{\partial z_{i}} \log (\mathcal{I})=\sum_{\substack{0 \leq j \leq 2 n+1 \\ j \neq i}} \frac{s_{i j}}{z_{i}-z_{j}}=0
$$

for $i=1, \ldots, n$. Or in terms of $\theta$-variables, from eq. (2.8), the scattering equations are

$$
\sum_{\substack{0 \leq j \leq n \\ j \neq i}}\left(s_{i j} \cot \left(\theta_{i j} / 2\right) \theta_{i \tilde{\jmath}}-s_{i \tilde{\jmath}} \tan \left(\theta_{i j} / 2\right)\right)=0
$$

where $\theta_{i j}=\theta_{i}-\theta_{j}$.

This number $2^{n-1} n$ ! can be recursively verified by taking soft limits [3]. However, here we use another strategy proposed by Cachazo, Mizera and Zhang [19]: each solution of scattering equation can be regarded as one stationary configuration of some particular particle system on some region of kinematic space $\mathcal{K}_{+}$.

In our case, the region of kinematic space $\mathcal{K}_{+}$is defined by taking all $s_{i j}$ and $s_{i \tilde{\jmath}}$ with $j \neq i$ to be positive, this particle system is a 2 -dimensional system and consists of $n+1$ pairs of particles connected by $n+1$ light rigid rods of unit length (see figure 1), where the centers of all rods are in the same position and each pair of particles connected by a rod have the same charge. If we denote the $i^{\text {th }}$ pair of particles as $i$ and $\tilde{i}$ and the charge product of particles $i$ and $j$ as $s_{i j}$, then the potential of this system is simply

$$
V(\theta)=-2 \sum_{0 \leq i<j \leq n}\left(s_{i j} \log \left|\sin \left(\theta_{i j} / 2\right)\right|+s_{i \tilde{\jmath}} \log \left|\cos \left(\theta_{i j} / 2\right)\right|\right) .
$$

Here we take no account of all contribution from particle pairs connected by the rigid rods, and the factor 2 arises from the fact of $s_{i j}=s_{\tilde{\imath} \tilde{\jmath}}$ and $s_{i \tilde{\jmath}}=s_{\tilde{\imath} j}$. Since all particles mutually repel on the region $\mathcal{K}_{+}$, it is obvious that the solutions of scattering equation eq. (2.11) are stationary points of the potential eq. (2.12) on this region, and all these solutions in terms of $\theta$-variables hence are real. 
Next, we take a simple counting of stationary configurations of this system. A naïve counting gives $2^{n+1}(n+1)$ ! since these rods have two distinct endpoints and different orderings of these rods correspond different stationary configurations. However the symmetry $\mathrm{U}(1) \rtimes \mathbb{Z}_{2}$ mentioned above will reduce this number to $\left(2^{n} n !\right) / 2$. (One rod can be taken as the reference point, and only one-half of all these orderings are indeed different since this is a central symmetric system.)

\section{Intersection numbers}

In this section, we consider the new geometric structures of the moduli space $\mathcal{M}_{n+1}^{\mathrm{c}}(\mathbb{R})$ after introducing the Koba-Nielsen factor.

Since Koba-Nielsen factor eq. (2.7) or eq. (2.8) is not a well-defined single-valued function on $\mathcal{M}_{n+1}^{\mathrm{c}}(\mathbb{R})$ whose branch points are the hyperplains defined by $z_{i}-z_{j}=0$ for all $0 \leq i<j \leq 2 n+1$, one should assign a branch of Koba-Nielsen factor on each chamber for the sake of certainty. Therefore, we can define twisted chambers $\Delta \otimes \mathcal{I}_{\Delta}$ as our basic objects, where $\Delta$ is a chamber and $\mathcal{I}_{\Delta}$ is a given branch of $\mathcal{I}$ on $\Delta$. The same consideration still works for the compactification of $\mathcal{M}_{n+1}^{\mathrm{c}}(\mathbb{R})$.

Generally, one can consider a twisted $m$-simplex $\sigma \otimes \mathcal{I}_{\sigma}$, where $\sigma$ is a topological $m$ simplex in $\mathcal{M}_{n+1}^{\mathrm{c}}(\mathbb{R})$, then there is a natural inherited boundary map $\partial_{\mathcal{I}}$ for any twisted simplex. For example, suppose $\sigma=\langle 01 \cdots m\rangle$ is a topological $m$-simplex, where $\langle 01 \cdots m\rangle$ is the standard notation [20], the traditional boundary map is

$$
\partial^{m} \sigma=\sum_{k=0}^{m}(-1)^{k}\langle 01 \cdots \hat{k} \cdots m\rangle
$$

and the boundary map $\partial_{\mathcal{I}}^{m}$ for any twisted simplex $\sigma \otimes \mathcal{I}_{\sigma}$ is

$$
\partial_{\mathcal{I}}^{m}\left(\sigma \otimes \mathcal{I}_{\sigma}\right)=\left.\sum_{k=0}^{m}(-1)^{k}\langle 01 \cdots \hat{k} \cdots m\rangle \otimes I_{\sigma}\right|_{\langle 01 \cdots \hat{k} \cdots m\rangle} .
$$

It's not hard to check that $\partial_{\mathcal{I}}^{m-1} \partial_{\mathcal{I}}^{m}=0$, so there's a natural homology group defined by

$$
H_{m}\left(\mathcal{M}_{n+1}^{\mathrm{c}}(\mathbb{R}), \partial_{\mathcal{I}}\right)=\frac{\operatorname{ker} \partial_{\mathcal{I}}^{m-1}}{\operatorname{im} \partial_{\mathcal{I}}^{m}}
$$

Kita and Yoshida $[18,21,22]$ investigated these objects for a general space and a general factor and developed a geometric theory according to these twisted simplexes and their integrals. In the twisted homology theory, a new intersection number of two twisted cycles is introduced and is a very useful tool to calculate some topological invariants. Loosely speaking, the intersection number is a pairing

$$
H_{n}\left(\mathcal{M}_{n+1}^{\mathrm{c}}(\mathbb{R}), \partial_{\mathcal{I}}\right) \times H_{n}\left(\mathcal{M}_{n+1}^{\mathrm{c}}(\mathbb{R}), \partial_{\mathcal{I}}\right) \rightarrow \mathbb{C}
$$

Especially, when factors in the Koba-Nielsen factor are all linear, they constructed manifestly twisted cycles in the section 2.3 and 3.2 of [18], which can also been found in [9] for a 
short introduction. Meanwhile, intersection numbers of these twisted cycles are calculated in the section 2.3.3 and 3.5.9 of [18].

Mizera [9] showed that the intersection numbers of associahedra (with chosen branches of Koba-Nielsen factor) in the moduli space form the inverse of the Kawai-Lewellen-Tye (KLT) kernel [23] which relates tree-level open string amplitudes and closed string amplitudes. What's more, under the limit $\alpha^{\prime} \rightarrow 0$, the elements of this inverse matrix will give amplitudes of bi-adjoint $\varphi^{3}$-theory $[6,24]$ which relate to the Cachazo-He-Yuan (CHY) formula. In this section, we consider intersection numbers of cyclohedra.

However, we will not go into the details of the whole geometric theory. Instead of defining intersection number step by step [18, 21, 22], we directly give a formula eq. (3.1) of two cyclohedra with the chosen branches $|\mathcal{I}|$.

Suppose $\Delta(\alpha)$ and $\Delta(\beta)$ are two cyclohedra, $K$ is their shared face with the lowest codimension $k$, and they are on the different side of $K$. Suppose $K=H_{1} \cap \cdots \cap H_{k}$ is the intersection of $k$ facets, and the chosen branches of $\mathcal{I}$ on $\Delta(\alpha)$ and $\Delta(\beta)$ is

$$
|\mathcal{I}|=f(z) \prod_{0 \leq i<j \leq n}\left|z_{i}-z_{j}\right|^{2 \alpha^{\prime} s_{i j}}\left|z_{i}-z_{\tilde{\jmath}}\right|^{2 \alpha^{\prime} s_{i \tilde{\jmath}}}
$$

where the factor $f(z)$ will not bother us since it never vanishes on $\mathcal{M}_{n+1}^{c}(\mathbb{R})$. Then the intersection number $[18,21,22]$ of $\Delta(\alpha)$ and $\Delta(\beta)$ is defined by

$$
\langle\Delta(\alpha), \Delta(\beta)\rangle:=\left(\frac{1}{2 \mathrm{i}}\right)^{k} \prod_{i=1}^{k} \frac{1}{\sin \left(\alpha^{\prime} \pi s_{H_{i}}\right)} \sum_{\text {faces } H \text { of } K} \frac{1}{d_{H}^{K}}
$$

where $s_{H_{i}}=2 s_{i_{1}, \ldots, i_{k}}=2 \sum_{i_{1} \leq p<q \leq i_{k}} s_{p q}$ for $H_{i}$ whose label is $\left(i_{1} \cdots i_{k}\right)$ and

$$
d_{\bigcap_{i} H_{i}}^{K}=\prod_{i}\left(\exp \left(2 \pi \mathrm{i} \alpha^{\prime} s_{H_{i}}\right)-1\right) \quad \text { and } \quad d_{K}^{K}=1 .
$$

For example,
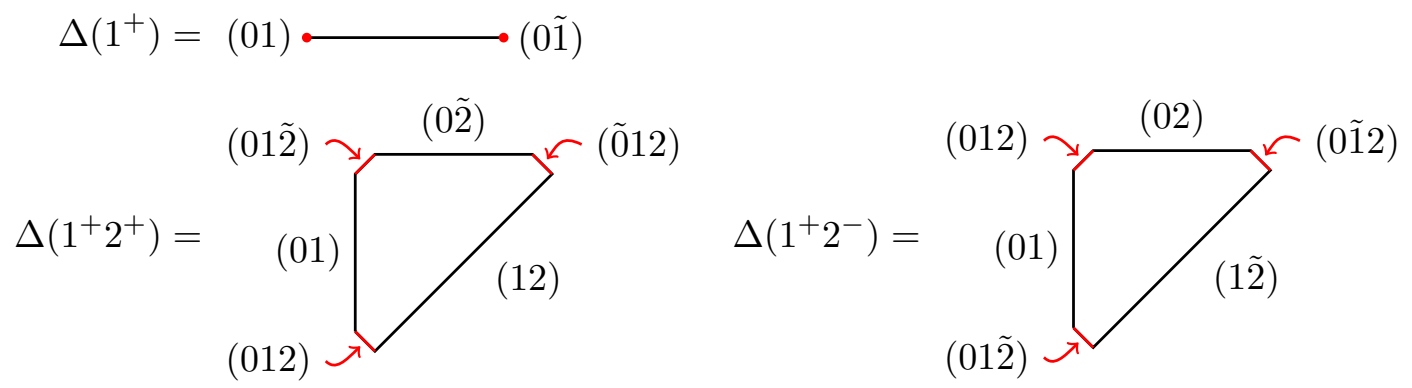

and the self intersection number of $\Delta\left(1^{+}\right)$is

$$
\begin{aligned}
\left\langle\Delta\left(1^{+}\right), \Delta\left(1^{+}\right)\right\rangle & =1+\frac{1}{\exp \left(2 \pi \mathrm{i} \alpha^{\prime} 2 s_{01}\right)-1}+\frac{1}{\exp \left(2 \pi \mathrm{i} \alpha^{\prime} 2 s_{0 \tilde{1}}\right)-1} \\
& =\frac{1}{2 \mathrm{i}}\left(\frac{1}{\tan \left(\alpha^{\prime} \pi 2 s_{01}\right)}+\frac{1}{\tan \left(\alpha^{\prime} \pi 2 s_{0 \tilde{1}}\right)}\right) .
\end{aligned}
$$


For $\Delta\left(1^{+} 2^{+}\right)$and $\Delta\left(1^{+} 2^{-}\right)$, they intersect at the face labeled by (01), so

$$
\begin{aligned}
\left\langle\Delta\left(1^{+} 2^{+}\right), \Delta\left(1^{+} 2^{-}\right)\right\rangle & =\frac{1}{2 \mathrm{i}} \frac{1}{\sin \left(\alpha^{\prime} \pi 2 s_{01}\right)}\left(1+\frac{1}{\exp \left(2 \pi \mathrm{i} \alpha^{\prime} 2 s_{012}\right)-1}+\frac{1}{\exp \left(2 \pi \mathrm{i} \alpha^{\prime} 2 s_{01 \tilde{2}}\right)-1}\right) \\
& =\frac{1}{(2 \mathrm{i})^{2}} \frac{1}{\sin \left(\alpha^{\prime} \pi 2 s_{01}\right)}\left(\frac{1}{\tan \left(\alpha^{\prime} \pi 2 s_{012}\right)}+\frac{1}{\tan \left(\alpha^{\prime} \pi 2 s_{01 \tilde{2}}\right)}\right) .
\end{aligned}
$$

Finally, define the 'KLT inverse matrix' by

$$
m_{\alpha^{\prime}}(\alpha \mid \beta)=(2 \mathrm{i})^{n}\langle\Delta(\alpha), \Delta(\beta)\rangle,
$$

where $n=\operatorname{dim} \Delta(\alpha)=\operatorname{dim} \Delta(\beta)$, and define

$$
m(\alpha \mid \beta)=\lim _{\alpha^{\prime} \rightarrow 0}\left(2 \alpha^{\prime} \pi\right)^{n} m_{\alpha^{\prime}}(\alpha \mid \beta) .
$$

For example,

$$
m\left(1^{+} 2^{+}, 1^{+} 2^{-}\right)=\frac{1}{s_{01}}\left(\frac{1}{s_{012}}+\frac{1}{s_{01 \tilde{2}}}\right) .
$$

In the next section, we will use CHY formula to calculate $m(\alpha \mid \beta)$, which gives another representation.

At the end of this section, we briefly comment on the dual of $H_{n}\left(\mathcal{M}_{n+1}^{c}(\mathbb{R}), \partial_{\mathcal{I}}\right)$, which is the cohomology group on $\mathcal{M}_{n+1}^{c}(\mathbb{R})$ defined by a flat connection

$$
\nabla=\mathrm{d}+\omega_{\mathcal{I}} \wedge
$$

with the property $\nabla^{2}=0$, where

$$
\omega_{\mathcal{I}}=\mathrm{d} \log \mathcal{I}=\sum_{i=1}^{n} E_{i} \mathrm{~d} z_{i}=\sum_{i=1}^{n} \sum_{\substack{0 \leq j \leq 2 n+1 \\ j \neq i}} \frac{s_{i j}}{z_{i}-z_{j}} \mathrm{~d} z_{i}
$$

is a well-defined single-valued form on $\mathcal{M}_{n+1}^{\mathrm{c}}(\mathbb{R})$. Let's denote these cohomology groups by $H^{\bullet}\left(\mathcal{M}_{n+1}^{\mathrm{c}}(\mathbb{R}), \omega_{\mathcal{I}}\right)$. As shown in $[25], H^{n}\left(\mathcal{M}_{n+1}^{\mathrm{c}}(\mathbb{R}), \omega_{\mathcal{I}}\right)$ is generated by d log forms

$$
\mathrm{d} \log \left(\frac{z_{i_{1}} \pm z_{j_{1}}}{z_{k_{1}} \pm z_{l_{1}}}\right) \wedge \cdots \wedge \mathrm{d} \log \left(\frac{z_{i_{n}} \pm z_{j_{n}}}{z_{k_{n}} \pm z_{l_{n}}}\right)
$$

where $0 \leq i_{r}, j_{r}, k_{r}, l_{r} \leq n$ for all $1 \leq r \leq n$, which should be invariant under $z \mapsto 1 / z$. Parke-Taylor forms eq. (2.2) belong to this class. Unlike the case of $\mathcal{M}_{0, n}(\mathbb{R})$, the independent Parke-Taylor forms can no longer generate the whole space $H^{n}\left(\mathcal{M}_{n+1}^{\mathrm{c}}(\mathbb{R}), \omega_{\mathcal{I}}\right)$. One has to consider more forms, for example,

$$
\mathrm{d} \log \left(\frac{z_{1}}{z_{0}}\right) \wedge \mathrm{d} \log \left(\frac{z_{2}}{z_{0}}\right)=\frac{\mathrm{d} z_{1} \wedge \mathrm{d} z_{2}}{z_{1} z_{2}} .
$$

It is invariant under the transformation $z \mapsto 1 / z$, and then belongs to $H^{2}\left(\mathcal{M}_{3}^{c}(\mathbb{R}), \omega_{\mathcal{I}}\right)$. However, it is not the canonical form of a cyclohedron anymore. 


\section{Kinematic cyclohedra and CHY formula}

In [6], Arkani-Hamed, Bai, He and Yan have constructed associahedra in kinematic space. They found a set of equations and inequalities of $X$-variables

$$
X_{i j}:=s_{i, i+1, \ldots, j-1}=\sum_{i \leq k<l \leq j-1} s_{k l}
$$

where the interior of an associahedron is given by $\left\{X_{i j}>0\right\}$ and the facet represented by $X_{i j}$ lays on the hyperplane defined by $X_{i j}=0$. They also found that the scattering equations can be interpreted as a map between worldsheet associahedra and kinematic associahedra. Here, we generalize these stories to cyclohedra.

\subsection{Kinematic cyclohedra}

Suppose $P_{n}$ is a $(2 n+2)$-gon, for the diagonal with vertices $i$ and $j$, we put a variable $X_{i j}$ on it, where labels live in $\mathbb{Z}_{2 n+2}$. Thanks to momentum conservation and identifications of $s_{i j}$ and $s_{\tilde{\imath} \tilde{\jmath}}, X_{i j}=X_{j i}=X_{\tilde{\imath} \tilde{\jmath}}$. Usually, it's more convenient to use $X_{\tilde{\imath} \tilde{\jmath}}=X_{i j}$ to represent a pair of centrally symmetric diagonals $(i, j)$ and $(\tilde{\imath}, \tilde{\jmath})$, where we think the longest diagonals $(i, \tilde{\imath})=(\tilde{\imath}, i)$ as 'degenerate' pairs. Therefore, there are $n(n+1)$ independent $X$-variables. The Mandelstam variables $s_{i j}$ are related to $X$-variables by

$$
-s_{i j}=X_{i j}+X_{i+1, j+1}-X_{i+1, j}-X_{i, j+1} .
$$

For future use, we generalize the above identities to

$$
-\sum_{i \leq a<j<k \leq b<l} s_{a b}=X_{i k}+X_{j l}-X_{j k}-X_{i l} .
$$

In order to construct a $n$-dimensional object from $n^{2}+n$ independent variables, we need $n^{2}$ constraints, which can be chosen as in [6]: let

$$
c_{i j}=-s_{i j}=X_{i, j}+X_{i+1, j+1}-X_{i, j+1}-X_{i+1, j}
$$

be positive constants for $0 \leq i<j-1<j \leq 2 n, i \neq n$ and $j \neq \tilde{n}$, where we require that $c_{i j}$ and $c_{\tilde{\imath} \tilde{\jmath}}$ are the same in addition, so the rank of these constraints is $n^{2}$. Our polytope $\mathcal{W}_{n}$ is given by inequalities

$$
X_{i j} \geq 0 \text { for all } 0 \leq i<j \leq 2 n+1,
$$

and setting eq. (4.2) to be positive constants. Such construction can also obtained from $C_{n}$ cluster algebra by using the method proposed in [26]. We will show that it is a cyclohedron after a short example.

For $n=2$, independent variables are $\left\{X_{02}, X_{0 \tilde{0}}, X_{0 \tilde{1}}, X_{1 \tilde{1}}, X_{1 \tilde{2}}, X_{2 \tilde{2}}\right\}$, and constraints are

$$
\begin{aligned}
X_{02}+X_{0 \tilde{1}}-X_{0 \tilde{0}} & =c_{02}, \\
X_{0 \tilde{0}}+X_{1 \tilde{1}}-2 X_{0 \tilde{1}} & =c_{0 \tilde{0}}, \\
X_{0 \tilde{1}}+X_{1 \tilde{2}}-X_{1 \tilde{1}} & =c_{0 \tilde{1}}, \\
X_{1 \tilde{1}}+X_{2 \tilde{2}}-2 X_{1 \tilde{2}} & =c_{1 \tilde{1}},
\end{aligned}
$$




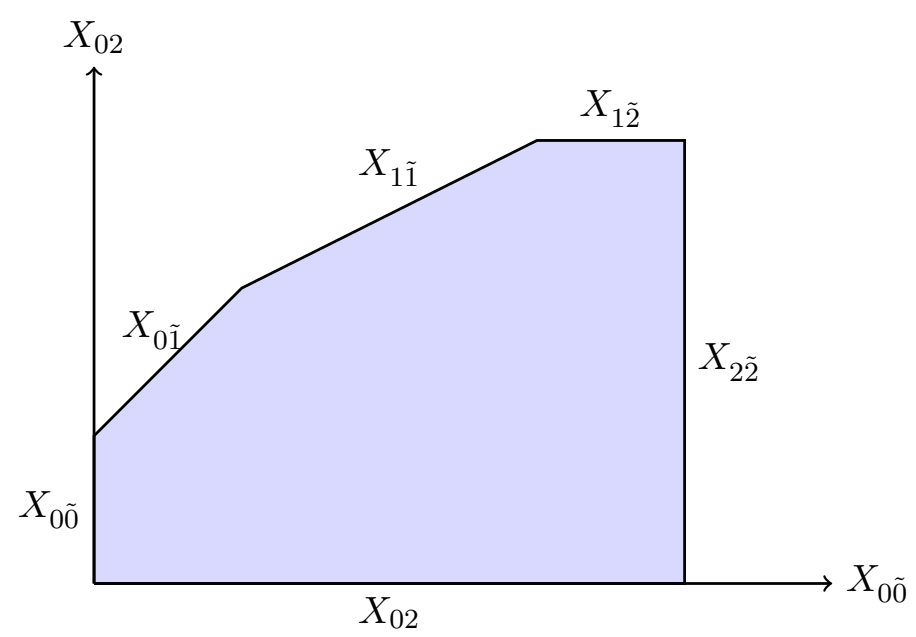

Figure 2. The 2-dimensional kinematic cyclohedron $\mathcal{W}_{2}$ after taking $c_{02}=c_{00}=c_{0 \tilde{1}}=c_{1 \tilde{1}}=1$.

where the factor 2 arises form identifications. Thus, $\mathcal{W}_{2}$ is the polytope defined by inequalities

$$
X_{02} \geq 0, \quad X_{0 \tilde{0}} \geq 0, \quad X_{0 \tilde{1}} \geq 0, \quad X_{1 \tilde{1}} \geq 0, \quad X_{1 \tilde{2}} \geq 0, \quad X_{2 \tilde{2}} \geq 0 .
$$

It's shown in figure 2 by using $X_{00}$ and $X_{02}$ as coordinates. As shown in figure $2, \mathcal{W}_{2}$ is a hexagon, that is a 2-dimensional cyclohedron. Now let's consider the $\mathcal{W}_{n}$ for a general $n$.

Proposition 2. $\mathcal{W}_{n}$ is the $n$-dimensional cyclohedron.

Proof. In our construction, every facet of the polytope $\mathcal{W}_{n}$ is given by setting the corresponding $X_{i j} \rightarrow 0$. However, it's impossible to set arbitrary two $X$-variables approaching to 0 together. Indeed, recall eq. (4.1) for $j \leq n$ and $l \leq \tilde{n}$,

$$
X_{j k}+X_{i l}=X_{i k}+X_{j l}-\sum_{i \leq a<j<k \leq b<l} c_{a b},
$$

the right-hand side of above equation is negative as $X_{i k}, X_{j l} \rightarrow 0$, while the left-hand side is always positive, so there is a contradiction. It follows that a lower dimensional boundary of $\mathcal{W}_{n}$ can not be reached by setting two intersecting diagonals to zero. Conversely, lower dimensional boundaries of $\mathcal{W}_{n}$ can only be reached by setting non-intersecting diagonals to zero. On the other hand, the dissections of $(2 n+2)$-gon in this way are always centrally symmetric since $X_{i j}=X_{\tilde{\imath}}$. Therefore, $\mathcal{W}_{n}$ is a $n$-dimensional cyclohedron according to definition 1.

It's very natural to construct other cyclohedra $\mathcal{W}\left(\sigma,\left\{s_{i}\right\}\right)$ in kinematic space. The data $\left\{\mathrm{s}_{1}, \mathrm{~s}_{2}, \ldots, \mathbf{s}_{n}\right\}$ and permutation $\sigma$ define an centrally symmetric ordering in $S^{2 n+2}$, and vice versa, for example, $\left(2^{+} 1^{-} 3^{+}\right) \leftrightarrow(02 \tilde{1} 3 \tilde{0} \tilde{2} 1 \tilde{3})$. Therefore, we identify these two notations of centrally symmetric orderings. Now, we can define $X$-variables in ordering $\alpha$ by setting

$$
X_{\alpha(i), \alpha(j)}=s_{\alpha(i), \alpha(i+1), \ldots, \alpha(j-1)}
$$



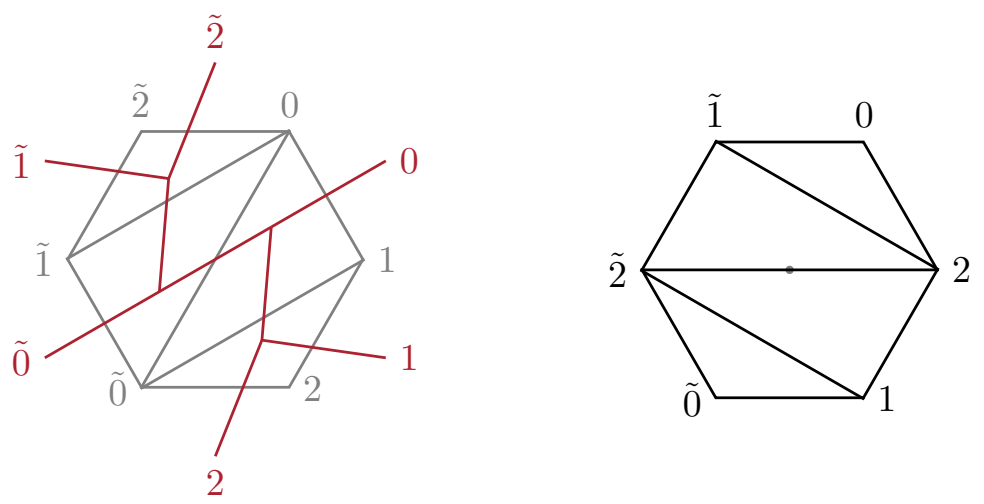

Figure 3. The dual graph of a triangulation (left) and a triangulation in the ordering (0210̃2 $\tilde{1})$ (right).

Then $\mathcal{W}_{n}(\alpha)$ in kinematic space is similarly given by setting

$$
H_{n}(\alpha)=\left\{c_{\alpha(i) \alpha(j)}=\text { positive constant } \mid 0 \leq i<j-1<j \leq 2 n, i \neq n \text { and } j \neq \tilde{n}\right\}
$$

and

$$
X_{\alpha(i), \alpha(j)} \geq 0 \quad \text { for all } i<j .
$$

Cyclohedra are simple polytopes, i.e. every vertex of $n$-dimensional cyclohedron $W_{n}$ is adjacent to $n$ facets. It's direct from the definition 1 of the cyclohedron. Since every centrally symmetric triangulation of a $(2 n+2)$-gon, which corresponds to a vertex of $W_{n}$, has $n$ pairs of centrally symmetric diagonals, which correspond to the adjacent facets of this vertex. Therefore the canonical form [14] of $\mathcal{W}_{n}$ is

$$
\Omega\left(\mathcal{W}_{n}\right)=\sum_{\operatorname{vertex} Z} \operatorname{sign}(Z) \bigwedge_{a=1}^{n} \mathrm{~d} \log X_{i_{a}^{Z}, j_{a}^{Z}},
$$

where each vertex $Z$ corresponds to a centrally symmetric triangulation of this $(2 n+2)$ gon, $X_{i_{a}^{Z}, j_{a}^{Z}}$ is the $X$-variable corresponding to the pair of diagonals $\left(i_{a}^{Z}, j_{a}^{Z}\right)$ and $\left(\tilde{\imath}_{a}^{Z}, \tilde{\jmath}_{a}^{Z}\right)$ in this triangulation, and $\operatorname{sign}(Z)$ is evaluated on the ordering of the facets in the wedge product to ensure $\Omega\left(\mathcal{W}_{n}\right)$ is a projective form $[6,14]$, for example,

$$
\begin{aligned}
\Omega\left(\mathcal{W}_{1}\right)= & \frac{\mathrm{d} X_{0 \tilde{0}}}{X_{0 \tilde{0}}}-\frac{\mathrm{d} X_{1 \tilde{1}}}{X_{1 \tilde{1}}}, \\
\Omega\left(\mathcal{W}_{2}\right)= & \frac{\mathrm{d} X_{02} \wedge \mathrm{d} X_{0 \tilde{0}}}{X_{02} X_{0 \tilde{0}}}-\frac{\mathrm{d} X_{02} \wedge \mathrm{d} X_{2 \tilde{2}}}{X_{02} X_{2 \tilde{2}}}+\frac{\mathrm{d} X_{1 \tilde{2}} \wedge \mathrm{d} X_{2 \tilde{2}}}{X_{1 \tilde{2}} X_{2 \tilde{2}}} \\
& -\frac{\mathrm{d} X_{1 \tilde{2}} \wedge \mathrm{d} X_{1 \tilde{1}}}{X_{1 \tilde{2}} X_{1 \tilde{1}}}+\frac{\mathrm{d} X_{0 \tilde{1}} \wedge \mathrm{d} X_{1 \tilde{1}}}{X_{0 \tilde{1}} X_{1 \tilde{1}}}-\frac{\mathrm{d} X_{0 \tilde{1}} \wedge \mathrm{d} X_{0 \tilde{0}}}{X_{0 \tilde{1}} X_{0 \tilde{0}}} .
\end{aligned}
$$

On the other aspect, every vertex of $\mathcal{W}_{n}$ also corresponds to the dual graph of each centrally symmetric triangulation of a $(2 n+2)$-gon, see figure 3 . If we further think dual graphs as planar Feynman diagrams with scalar propagators, they share the same poles 
with the term corresponding to the vertex $Z$ in eq. (4.5)

$$
\bigwedge_{a=1}^{n} \mathrm{~d} \log X_{i_{a}^{Z}, j_{a}^{Z}}=\frac{\bigwedge_{a=1}^{n} \mathrm{~d} X_{i_{a}^{Z}, j_{a}^{Z}}}{\prod_{a=1}^{n} X_{i_{a}^{Z}, j_{a}^{Z}}}
$$

For example, for the triangulation in the left-hand side of figure 3, poles of this planar Feynman diagram are $s_{12}=s_{\tilde{1} \tilde{2}}$ and $s_{012}=s_{\tilde{0} \tilde{1} \tilde{2}}$, and the corresponding scattering form is

$$
\frac{\mathrm{d} X_{02} \wedge \mathrm{d} X_{03}}{X_{02} X_{03}}=\frac{\mathrm{d} s_{01} \wedge \mathrm{d} s_{012}}{s_{01} s_{012}} .
$$

Note that, $1 /\left(s_{01} s_{012}\right)$ is not the amplitude determined by this Feynman diagram. Besides, we can even $\operatorname{read} \operatorname{sign}(Z)$ from this viewpoint. Indeed, if $Z$ and $Z^{\prime}$ are full centrally symmetric triangulations related by a mutation of a pair of diagonals or a long diagonal, then $\operatorname{sign}(Z)=-\operatorname{sign}\left(Z^{\prime}\right)$.

We can extend this viewpoint of dual graph to other orderings. For an ordering $\alpha$, define $\operatorname{csp}(\alpha)$ to be the set of all dual graphs of full centrally symmetric triangulations in the ordering $\alpha$ (see the right-hand side of figure 3 for an example). If a planar graph $g$ belongs to $\operatorname{csp}(\alpha)$, we say that this graph is compatible with $\alpha$. Therefore,

$$
\Omega\left(\mathcal{W}_{n}(\alpha)\right)=\sum_{g \in \operatorname{csp}(\alpha)} \operatorname{sign}(g) \bigwedge_{a=1}^{n} \mathrm{~d} \log X_{i_{a}^{g}, j_{a}^{g}}
$$

Now for any graph $g$, we can pullback it to the subspace $H_{n}(\alpha)$, then $[6]$

$$
\left.\left(\operatorname{sign}(g) \bigwedge_{a=1}^{n} \mathrm{~d} X_{i_{a}^{g}, j_{a}^{g}}\right)\right|_{H(\alpha)}= \begin{cases}\mathrm{d}^{n} X(\alpha) & \text { if } g \in \operatorname{csp}(\alpha) \\ 0 & \text { otherwise }\end{cases}
$$

where we assume that $\operatorname{sign}(g)$ for a compatible graph in different orderings are equal. One can check that $\mathrm{d}^{n} X(\alpha)$ is independent of the choice of $g$, so it's the volume form of $\mathcal{W}_{n}(\alpha)$.

Now we define $m(\alpha \mid \beta)$ by the pullback of $\Omega\left(\mathcal{W}_{n}(\alpha)\right)$ to the subspace $H_{n}(\beta)$ :

$$
\left.\Omega\left(\mathcal{W}_{n}(\alpha)\right)\right|_{H_{n}(\beta)}=: m(\alpha \mid \beta) \mathrm{d}^{n} X(\beta),
$$

or more concretely by eq. (4.6),

$$
m(\alpha \mid \beta)=\sum_{g \in \operatorname{csp}(\alpha) \cap \operatorname{csp}(\beta)} \prod_{a=1}^{n} \frac{1}{X_{i_{a}^{g}, j_{a}^{g}}} .
$$

To this point, one can easily verify eq. (3.2) by checking the combinatoric structure of both sides.

\subsection{Scattering equations as a map between cyclohedra}

Up to now, we have constructed cyclohedra in moduli space and kinematic space. Here we claim that the connection between these two cyclohedra is given by the so-called scattering equation map [6] which is the solution of $X_{i j}$ of the scattering equations eq. (2.10). 
Recall that, in our construction of kinematic cyclohedra,

$$
s_{01}, s_{12}, \ldots, s_{n-1, n}, s_{0 \tilde{n}}, s_{1 \tilde{n}}, \ldots, s_{n \tilde{n}}
$$

are not constants. We can eliminate $s_{i \tilde{n}}$ by momentum conservation, then scattering equations eq. (2.10) are linear equations of $\left\{s_{i, i+1} \mid 0 \leq i \leq n-1\right\}$, whose solution is

$$
s_{k, k+1}=\sum_{0 \leq i \leq k<k+1 \leq j \leq 2 n} \frac{\sin \left(\theta_{i \tilde{n}} / 2\right) \sin \left(\theta_{j \tilde{n}} / 2\right) \sin \left(\theta_{k, k+1} / 2\right)}{\sin \left(\theta_{i j} / 2\right) \sin \left(\theta_{k \tilde{n}} / 2\right) \sin \left(\theta_{k+1, \tilde{n}} / 2\right)} c_{i j}
$$

for $k=0, \ldots, n-1$. Eq. (4.8) defines a map $\varphi: z \mapsto X$ from moduli space to kinematic space. It's direct from eq. (4.8) that $X_{k, k+2}=s_{k, k+1}>0$ when $0<\theta_{1}<\cdots<\theta_{n}<\pi$. The other $X_{i j}$ 's can also be computed by the definition of $X$-variables and one can check that all these $X$-variables are positive. Therefore, this map further maps the worldsheet cyclohedra $W_{n}(\mathrm{id},\{+\})$ into the kinematic cyclohedra $\mathcal{W}_{n}$.

What's more, the scattering equation map is a map between the interiors of these two cyclohedra. It's equivalent to say that $\varphi(z)$ is in the boundary of $\mathcal{W}_{n}$ if and only if $z$ is in the boundary of $W_{n}(\mathrm{id},\{+\})$. This is straightforward from the scattering equation map eq. (4.8) or scattering equations eq. (2.10). For example, suppose $\theta_{p}=\theta_{r}+t x_{p}$ for $r<p \leq s$, when $t$ goes to 0 ,

$$
s_{k, k+1} \rightarrow \sum_{i=r}^{k-1} \frac{x_{k+1}-x_{k}}{x_{k+1}-x_{i}} c_{i, k+1}+\sum_{i=r}^{k} \sum_{j=k+2}^{s} \frac{x_{k+1}-x_{k}}{x_{j}-x_{i}} c_{i j},
$$

for $r \leq k<s$. Therefore,

$$
\sum_{k=r}^{s-1} s_{k, k+1} \stackrel{t \rightarrow 0}{\longrightarrow} \sum_{i=r}^{s} \sum_{j=i+2}^{s} c_{i j}
$$

and then

$$
X_{r, s+1}=s_{r, \ldots, s}=\sum_{k=r}^{s-1} s_{k, k+1}-\sum_{i=r}^{s} \sum_{j=i+2}^{s} c_{i j} \rightarrow 0 .
$$

Conversely, one can use eq. (4.1) to rewrite eq. (2.10) in $X$ and $z$-variables, then when $X_{i j}$ goes to zero, the corresponding $z_{k}-z_{i}$ goes to zero for $i<k<j$. Actually, it does not depend on the condition $c_{i j}>0$ at all. We conjecture that $\varphi$ is a one-to-one map after imposing these conditions.

The pushforward [6] of scattering equation map connects the canonical forms of these two cyclohedra, i.e.

$$
\sum_{\text {sol. } z} \mathrm{PT}_{n}\left(1^{+} \cdots n^{+}\right)=\left.\Omega\left(\mathcal{W}_{n}(\mathrm{id})\right)\right|_{H_{n}(\mathrm{id})}=m(\mathrm{id} \mid \mathrm{id}) \mathrm{d}^{n} X(\mathrm{id}),
$$

where $z$ are solutions of scattering equations. One can consider other ordering pairs $\alpha, \beta$ and the scattering equation map $\varphi^{\alpha}$, then

$$
\sum_{\text {sol. } z} \mathrm{PT}_{n}(\beta)=\left.\Omega\left(\mathcal{W}_{n}(\alpha)\right)\right|_{H_{n}(\beta)}=m(\alpha \mid \beta) \mathrm{d}^{n} X(\beta) .
$$


We can rewrite this pushforward in terms of delta function:

$$
\begin{aligned}
m(\alpha \mid \beta) & =\int \mathrm{PT}_{n}(\beta) \prod_{a=1}^{n} \delta\left(X_{\alpha\left(i_{a}\right), \alpha\left(j_{a}\right)}-\varphi_{a}^{\alpha}(z)\right) \\
& =\int \mathrm{PT}_{n}(\beta) \mathrm{PT}_{n}(\alpha) \prod_{a=1}^{n} \delta\left(E_{a}\right) \\
& =: m_{\mathrm{CHY}}(\alpha \mid \beta) .
\end{aligned}
$$

\section{$5 \quad Z$-integrals on the moduli space $\mathcal{M}_{n+1}^{\mathrm{c}}(\mathbb{R})$}

It is the place to consider some basic prototypes of string-like integrals on such moduli space since Parke-Taylor factors and Koba-Nielsen factors on this space have been defined. By analogy with the story in $\mathcal{M}_{0, n}$, it's natural to define the so-called ' $Z$-integrals' [15] on $\mathcal{M}_{n+1}^{\mathrm{c}}(\mathbb{R})$ as

$$
Z_{\alpha}(\beta)=\int_{\mathfrak{C}(\alpha)} \operatorname{PT}(\beta) \prod_{0 \leq i<j \leq n}\left(-\frac{\left(z_{\alpha(i)}-z_{\alpha(j)}\right)^{2}}{4 z_{\alpha(i)} z_{\alpha(j)}}\right)^{\alpha^{\prime} s_{i j}}\left(\frac{\left(z_{\alpha(i)}+z_{\alpha(j)}\right)^{2}}{4 z_{\alpha(i)} z_{\alpha(j)}}\right)^{\alpha^{\prime} s_{i \tilde{\jmath}}},
$$

where $\mathrm{PT}(\beta)$ is the Parke-Taylor factor defined by eq. (2.4) with respect to the ordering $\beta$, $\mathfrak{C}(\alpha)$ denotes the integration ordering on a circle which is defined by $\theta_{\alpha(0)}<\theta_{\alpha(1)}<\cdots<$ $\theta_{\alpha(n)}<\theta_{\alpha(\tilde{0})}$ (recall that $\theta_{i}=\arg \left(z_{i}\right)$ ), and the form of Koba-Nielsen factor is directly derived from eq. (2.8) by a change of variables and hence ensure its reality. Obviously, these integrals satisfy KK-like relations

$$
\begin{aligned}
Z_{\alpha}\left(1^{+} 2^{\mathrm{s}_{2}} \ldots n^{\mathrm{s}_{n}}\right)+ & Z_{\alpha}\left(2^{\mathrm{s}_{2}} 1^{+} \ldots n^{\mathrm{s}_{n}}\right)+\cdots+Z_{\alpha}\left(2^{\mathrm{s}_{2}} \ldots n^{\mathrm{s}_{n}} 1^{+}\right)+ \\
& Z_{\alpha}\left(1^{-} 2^{\mathrm{s}_{2}} \ldots n^{\mathrm{s}_{n}}\right)+Z_{\alpha}\left(2^{\mathrm{s}_{2}} 1^{-} \ldots n^{\mathrm{s}_{n}}\right)+\cdots+Z_{\alpha}\left(2^{\mathrm{s}_{2}} \ldots n^{\mathrm{s}_{n}} 1^{-}\right)=0 .
\end{aligned}
$$

In what follows, we will limit ourselves to the case of $\mathfrak{C}(012 \cdots n)$ since all the other cases just differ by a relabelling of indices, and the subscript $\alpha$ on $Z$ will also be omitted since the integration ordering has been fixed. Further more, we fix $z_{0}$ as 1 (then $\theta_{0}=0$ and $\left.\theta_{\tilde{0}}=\pi\right)$ to remove the $\mathrm{U}(1)$ redundancy.

In this section, we will take $\alpha^{\prime}$-expansion for two simple examples of integrals (5.1) to give a taste of number theoretical properties. We will see, not only multiple zeta values, but also alternating Euler sums appear in the $\alpha^{\prime}$-expansion. Before proceeding, it's worth mentioning an important limit of Koba-Nielson factor (2.9)

$$
\mathcal{I}^{\prime}=\prod_{0 \leq i<j \leq n}\left(\frac{\left(x_{i}-x_{j}\right)^{2}}{\left(1+x_{i}^{2}\right)\left(1+x_{j}^{2}\right)}\right)^{s_{i j}}=\prod_{0 \leq i<j \leq n}\left(x_{i}-x_{j}\right)^{2 \alpha^{\prime} s_{i j}} \prod_{i=0}^{n}\left|x_{i}-\mathrm{i}\right|^{2 \alpha^{\prime} s_{i \tau}}
$$

which is obtained by taking all $s_{i \tilde{\jmath}}$ with $i \neq j$ to be vanish, where $s_{i \tilde{\imath}}$ now is $-\sum_{j=0}^{n} s_{i j}$. Note that, eq. (5.3) is proportional to the Koba-Nielsen factor for $n+1$ open strings and one closed string [27]

$$
\mathcal{I}_{n+1,1}=|z-\bar{z}|^{\alpha^{\prime} q_{\|}^{2}} \prod_{i<j}\left|x_{i}-x_{j}\right|^{2 \alpha^{\prime} p_{i} \cdot p_{j}} \prod_{i}\left|x_{i}-z\right|^{2 \alpha^{\prime} p_{i} \cdot q}
$$


under the gauge fixing $z=\mathrm{i}$. However, some $Z$-integrals we defined don't have a good behaviour under this limit, and a comprehensive treatment of such integrals lie somewhat out the main line of this paper, we leave it to the future work.

Let us first consider the simplest case, $n=1$. In this case, there is actually only one kind of Parke-Taylor factor since $\mathrm{PT}\left(1^{+}\right)=-\mathrm{PT}\left(1^{-}\right)$, and the $Z$-integral is simply

$$
\begin{aligned}
Z\left(1^{+}\right) & =\int_{0}^{\infty} \frac{\mathrm{d} x_{1}}{-2 x_{1}}\left(\frac{x_{1}^{2}}{1+x_{1}^{2}}\right)^{\alpha^{\prime} s_{01}}\left(\frac{1}{1+x_{1}^{2}}\right)^{\alpha^{\prime} s_{0 \tilde{1}}} \\
& =-\frac{\Gamma\left(\alpha^{\prime} s_{01}\right) \Gamma\left(\alpha^{\prime} s_{0 \tilde{1}}\right)}{4 \Gamma\left(\alpha^{\prime} s_{01}+\alpha^{\prime} s_{0 \tilde{1}}\right)}
\end{aligned}
$$

Here the $x$-parameterization is used. The result is the usual Beta function, hence the $\alpha^{\prime}-$ expansion can be trivially obtained. The reader can check this result is divergent under the limit $s_{0 \tilde{1}} \rightarrow 0$.

The first nontrivial case is $n=2$. In this case, the result of integral can no longer be expressed as special functions we are familiar with, like the usual hypergeometric function appearing in string tree amplitudes. Thus, we turn to seek its $\alpha^{\prime}$-expansion. As pointed out in the paper [28], the obstacle to this expansion is singularities in Mandelstam variables pole. Putting it manifestly, the leading order of the integral (5.1), which can be obtained as a CHY formula

$$
Z_{\alpha}(\beta) \sim\left(\alpha^{\prime}\right)^{-n} m(\alpha \mid \beta)+\cdots,
$$

can not appear in a Taylor expansion in $\alpha^{\prime}$. An approach to this problem is pole subtractions introduced in [29]. The basic idea of this method is to subtract the integrand by several terms which can be integrated out more easily and contain the pole contributions. There are four $Z$-integrals for $n=2$ which are related by

$$
Z\left(1^{+} 2^{+}\right)=-Z\left(2^{+} 1^{+}\right)-Z\left(1^{-} 2^{+}\right)-Z\left(2^{+} 1^{-}\right),
$$

and the pole subtraction for each term on the R.H.S. is easier than $Z\left(1^{+} 2^{+}\right)$, which can be seen from leading contributions of these integrals. Further more, $Z\left(1^{-} 2^{+}\right)$and $Z\left(2^{+} 1^{-}\right)$ can be obtained from $Z\left(2^{+} 1^{+}\right)$through shifting particle indices by -1 and +1 , respectively. Thus, the remaining task is to attack the integral $Z\left(2^{+} 1^{+}\right)$.

We omit the detail of pole subtractions since this process is technical and tedious. The $\alpha^{\prime}$-expansion of remainder term after pole subtractions can be obtained by using the Maple program HyperInt [30] with an appropriate choice of the integration ordering. The result is simply

$$
\begin{aligned}
-Z\left(2^{+} 1^{+}\right)= & \frac{1}{4 \alpha^{\prime 2}}\left(\frac{1}{s_{12} s_{012}}+\frac{1}{s_{12} s_{\tilde{0} 12}}\right)+\frac{\zeta_{2}}{4}\left(4-\frac{4 s_{\tilde{0} 2}}{s_{\tilde{0} 12}}-\frac{s_{\tilde{0} 12}+s_{012}}{s_{12}}-\frac{4 s_{01}}{s_{012}}\right) \\
+ & \frac{\alpha^{\prime} \zeta_{3}}{2}\left(\frac{\left(s_{\tilde{0} 12}+s_{012}\right)^{2}}{2 s_{12}}+\frac{4 s_{01}\left(s_{12}+s_{01}\right)}{s_{012}}+\frac{4 s_{\tilde{0} 2}\left(s_{12}+s_{\tilde{0} 2}\right)}{s_{\tilde{0} 12}}\right. \\
& \left.-2\left(2 s_{12}+s_{\tilde{0} 2}+s_{01}+s_{012}\right)\right)+O\left(\alpha^{\prime 2}\right) .
\end{aligned}
$$


The next and higher orders of $\alpha^{\prime}$ are too long to record here, thus we just report the numbers appearing in the $\alpha^{\prime}$-expansion:

$$
\begin{array}{ll}
\alpha^{\prime 2}: & \zeta_{2}^{2}, \\
\alpha^{\prime 3}: & \zeta_{2} \zeta_{3}, \zeta_{5} \\
\alpha^{\prime 4}: & \zeta_{1,-3} \zeta_{2}, \zeta_{2}^{3}, \zeta_{3}^{2}
\end{array}
$$

where the convention for MZVs is

$$
\zeta_{n_{1}, \ldots, n_{r}}=\mathrm{Li}_{\left|n_{1}\right|, \ldots,\left|n_{r}\right|}\left(\frac{n_{1}}{\left|n_{1}\right|}, \ldots, \frac{n_{r}}{\left|n_{r}\right|}\right)
$$

with the usual Goncharov's polylogarithms $\operatorname{Li}_{n_{1}, \ldots, n_{r}}\left(z_{1}, \ldots, z_{r}\right)$ of weight $n=n_{1}+\cdots+$ $n_{r}[31]$.

Here we briefly remark the $\alpha^{\prime}$-expansion of $Z\left(2^{+} 1^{+}\right)$on the following points:

(i) We do this integral beginning with $x$-parameterization. Then the result is not as simple as in eq. (5.8), where the numerical coefficients involving the letters $\{ \pm \mathrm{i}\}$ appear. ('Letters' mean the possible numbers appearing in iterated integral which can be translated into the input of polylogarithms.)

(ii) However, a huge simplification of the result is realized by a series of changes of integration variables, $\left\{y_{1}=x_{1}^{2}, y_{2}=x_{1} x_{2}\right\}$ and $\left\{t_{i}=y_{i} /\left(1+y_{i}\right) \mid i=1,2\right\}$. In terms of the variables $\left\{t_{i}\right\}$, the Koba-Nielsen factor can be written as

$$
\mathcal{I}=t_{1}^{\alpha^{\prime} s_{01 \tilde{2}}}\left(1-t_{1}\right)^{\alpha^{\prime} s_{0 \tilde{2} 2}} t_{2}^{2 \alpha^{\prime} s_{02}}\left(1-t_{2}\right)^{\alpha^{\prime} s_{0 \tilde{2}}}\left(t_{1}-2 t_{1} t_{2}+t_{2}^{2}\right)^{\alpha^{\prime} s_{2} \tilde{2}}\left(t_{2}-t_{1}\right)^{2 \alpha^{\prime} s_{12}}
$$

In its present form, the linear reducibility of $\alpha^{\prime}$-expansion of the Koba-Nielsen factor is manifest, then a similar analysis as in the section 6 of [32] (also see [33, 34]) will tell us that only the letters $\{-1,0,1\}$ can appear, namely, the numbers appearing in the $\alpha^{\prime}$-expansion can only be the usual multiple zeta values and alternating Euler sums, like $\log 2$ and $\zeta_{1,-3}$ and so on. Note that, it is the introduction of $y$-variables that makes the linear reducibility better.

(iii) There is no contribution of order $\alpha^{\prime-1}$ although the number $\log 2=\zeta_{-1}$ of weight 1 does exist.

\section{Outlook}

In this article, we study a new moduli space $\mathcal{M}_{n+1}^{\mathrm{c}}$ and the corresponding kinematic space via two mathematic tools, intersection theory and positive geometry. We have shown that $\mathcal{M}_{n+1}^{\text {c }}(\mathbb{R})$ is tiled by $2^{n-1} n$ ! copies of the cyclohedron $W_{n}$, and each of which corresponds to a stationary point of the system with $n+1$ pairs of particles on a circle. We construct the 'inverse KLT matrix' for this case by considering intersection numbers of twisted cycles. At the same time, we construct another cyclohedron $\mathcal{W}_{n}$ in kinematic space and prove the scattering equations provide a map from $W_{n}$ to $\mathcal{W}_{n}$. It seems that everything works well 
as in the old story $\mathcal{M}_{0, n}$. However, there are several problems we have not taken up yet, for instance, how to triangulate the kinematic cyclohedra themselves as in [35], from which we expect to obtain a recursion relation for $m(\alpha \mid \beta)$.

Meanwhile, there are two aspects that are obviously different from the case for $\mathcal{M}_{0, n}$. One is the mismatch between the number of independent Parke-Taylor factors, which is conjectured to be $(2 n-1)$ !!, and the number of solutions of scattering equations, which is proved to be $(2 n) ! ! / 2$. The reason for this mismatch, as pointed out at the end of section 3 , is that there are $\mathrm{d} \log$ forms beyond Parke-Taylor forms in the whole twisted cohomology group. One consequence is the lack of BCJ-like relations as in $[3,36]$ which first appear in gauge theory amplitudes and is crucial for the color-kinematics duality [37]. Therefore, an important and interesting question is how to find other $\mathrm{d} \log$ forms to complete the basis of the whole twisted cohomology group.

Another direction is obviously the $Z$-integrals we defined in section 5 , which can be understood as a special case of a very rich class of 'integrals over cluster associahedron' [38] and will be explored (together with many other topics) further. We just showed the $\alpha^{\prime}$ expansion for two simple examples, however, new numerical coefficients compared to the $Z$-integrals over $\mathcal{M}_{0, n}$ have started to appear. So, two natural questions are which kind of numbers can appear in the $\alpha^{\prime}$-expansion of such integrals, and do these $\alpha^{\prime}$-expansions have the similar structure as in [39-41]? Meanwhile, it is also interesting to find the $\alpha^{\prime}$-expansion without integrating as in $[15,42]$.

Apart from all questions mentioned above, another interesting question is the connection with the scattering of open strings and one closed string. Although the corresponding Koba-Nielsen factor emerges through a specific limit of Mandelstam variables, other ingredients of such string integrals are still missing in our treatment. What's more, it's important to find the connection between the moduli spaces of such string integrals, i.e. one puncture in the interior and $n+1$ punctures at the boundary of a disk, and our case. The connection between these two moduli spaces may be a clue of all missing parts.

\section{Acknowledgments}

The original idea for this work came from the study of positive geometries, canonical forms and integrals related to cluster associahedra, by Nima Arkani-Hamed, Song He and Hugh Thomas, to whom we are grateful for suggesting the project, sharing ideas and many valuable inputs. We thank Gongwang Yan for collaborations in an early stage of the project. We also thank Giulio Salvatori for inspiring discussions, and Erik Panzer for his kind instruction on the nice program HyperInt.

Open Access. This article is distributed under the terms of the Creative Commons Attribution License (CC-BY 4.0), which permits any use, distribution and reproduction in any medium, provided the original author(s) and source are credited. 


\section{References}

[1] E. Witten, Perturbative gauge theory as a string theory in twistor space, Commun. Math. Phys. 252 (2004) 189 [hep-th/0312171] [INSPIRE].

[2] R. Roiban, M. Spradlin and A. Volovich, On the tree level S matrix of Yang-Mills theory, Phys. Rev. D 70 (2004) 026009 [hep-th/0403190] [INSPIRE].

[3] F. Cachazo, S. He and E.Y. Yuan, Scattering equations and Kawai-Lewellen-Tye orthogonality, Phys. Rev. D 90 (2014) 065001 [arXiv: 1306.6575] [INSPIRE].

[4] F. Cachazo, S. He and E.Y. Yuan, Scattering of Massless Particles in Arbitrary Dimensions, Phys. Rev. Lett. 113 (2014) 171601 [arXiv:1307.2199] [INSPIRE].

[5] F. Cachazo, S. He and E.Y. Yuan, Scattering of Massless Particles: Scalars, Gluons and Gravitons, JHEP 07 (2014) 033 [arXiv:1309.0885] [INSPIRE].

[6] N. Arkani-Hamed, Y. Bai, S. He and G. Yan, Scattering Forms and the Positive Geometry of Kinematics, Color and the Worldsheet, JHEP 05 (2018) 096 [arXiv:1711.09102] [INSPIRE].

[7] S. He, G. Yan, C. Zhang and Y. Zhang, Scattering Forms, Worldsheet Forms and Amplitudes from Subspaces, JHEP 08 (2018) 040 [arXiv: 1803.11302] [INSPIRE].

[8] G. Salvatori, 1-loop Amplitudes from the Halohedron, arXiv:1806.01842 [INSPIRE].

[9] S. Mizera, Combinatorics and Topology of Kawai-Lewellen-Tye Relations, JHEP 08 (2017) 097 [arXiv: 1706. 08527] [INSPIRE].

[10] S. Mizera, Scattering Amplitudes from Intersection Theory, Phys. Rev. Lett. 120 (2018) 141602 [arXiv: 1711.00469] [INSPIRE].

[11] S. Mizera, Inverse of the String Theory KLT Kernel, JHEP 06 (2017) 084 [arXiv: 1610.04230] [INSPIRE].

[12] J.D. Stasheff, Homotopy associativity of h-spaces. I, Trans. Am. Math. Soc. 108 (1963) 275.

[13] J.D. Stasheff, Homotopy associativity of h-spaces. II, Trans. Am. Math. Soc. 108 (1963) 293.

[14] N. Arkani-Hamed, Y. Bai and T. Lam, Positive Geometries and Canonical Forms, JHEP 11 (2017) 039 [arXiv:1703.04541] [INSPIRE].

[15] C.R. Mafra and O. Schlotterer, Non-abelian Z-theory: Berends-Giele recursion for the $\alpha^{\prime}$-expansion of disk integrals, JHEP 01 (2017) 031 [arXiv:1609.07078] [INSPIRE].

[16] S. Devadoss, Tessellations of moduli spaces and the mosaic operad, Contemp. Math. 239 (1999) 91 [math/9807010].

[17] L. Devadoss, A space of cyclohedra, Discrete Comput. Geom. 29 (2002) 61 [math/0102166].

[18] K. Aomoto, M. Kita, T. Kohno and K. Iohara, Theory of hypergeometric functions, Springer (2011).

[19] F. Cachazo, S. Mizera and G. Zhang, Scattering Equations: Real Solutions and Particles on a Line, JHEP 03 (2017) 151 [arXiv: 1609.00008] [INSPIRE].

[20] M. Nakahara, Geometry, topology and physics, CRC Press (2003).

[21] M. Kita and M. Yoshida, Intersection theory for twisted cycles, Math. Nachr. 166 (1994) 287.

[22] M. Kita and M. Yoshida, Intersection theory for twisted cycles II - Degenerate arrangements, Math. Nachr. 168 (1994) 171. 
[23] H. Kawai, D.C. Lewellen and S.H.H. Tye, A Relation Between Tree Amplitudes of Closed and Open Strings, Nucl. Phys. B 269 (1986) 1 [INSPIRE].

[24] H. Frost, Biadjoint scalar tree amplitudes and intersecting dual associahedra, JHEP 06 (2018) 153 [arXiv: 1802.03384] [INSPIRE].

[25] H. Esnault, V. Schechtman and E. Viehweg, Cohomology of local systems on the complement of hyperplanes, Invent. Math. 109 (1992) 557.

[26] V. Bazier-Matte, G. Douville, K. Mousavand, H. Thomas and E. Yıldırım, Abhy associahedra and newton polytopes of $f$-polynomials for finite type cluster algebras, arXiv:1808.09986.

[27] S. Stieberger, Open 8 Closed vs. Pure Open String Disk Amplitudes, arXiv:0907.2211 [INSPIRE].

[28] J. Broedel, O. Schlotterer and S. Stieberger, Polylogarithms, Multiple Zeta Values and Superstring Amplitudes, Fortsch. Phys. 61 (2013) 812 [arXiv:1304.7267] [INSPIRE].

[29] O. Schlotterer and O. Schnetz, Closed strings as single-valued open strings: A genus-zero derivation, J. Phys. A 52 (2019) 045401 [arXiv: 1808.00713] [INSPIRE].

[30] E. Panzer, Algorithms for the symbolic integration of hyperlogarithms with applications to Feynman integrals, Comput. Phys. Commun. 188 (2015) 148 [arXiv: 1403.3385] [InSPIRE].

[31] A.B. Goncharov, Multiple polylogarithms, cyclotomy and modular complexes, Math. Res. Lett. 5 (1998) 497 [arXiv:1105.2076] [INSPIRE].

[32] F.C.S. Brown, Multiple zeta values and periods of moduli spaces $\mathfrak{M}_{0, n}$, Annales Sci. Ecole Norm. Sup. 42 (2009) 371 [math/0606419].

[33] F.C.S. Brown, On the periods of some Feynman integrals, arXiv:0910.0114 [INSPIRE].

[34] E. Panzer, Feynman integrals and hyperlogarithms, Ph.D. Thesis, Humboldt U., Berlin, Inst. Math. (2015) [arXiv: 1506.07243] [INSPIRE].

[35] S. He and Q. Yang, An Etude on Recursion Relations and Triangulations, arXiv: 1810.08508 [INSPIRE].

[36] F. Cachazo, Fundamental BCJ Relation in $N=4$ SYM From The Connected Formulation, arXiv: 1206.5970 [INSPIRE].

[37] Z. Bern, J.J.M. Carrasco and H. Johansson, New Relations for Gauge-Theory Amplitudes, Phys. Rev. D 78 (2008) 085011 [arXiv:0805.3993] [INSPIRE].

[38] N. Arkani-Hamed, S. He and H. Thomas, to appear.

[39] O. Schlotterer and S. Stieberger, Motivic Multiple Zeta Values and Superstring Amplitudes, J. Phys. A 46 (2013) 475401 [arXiv:1205.1516] [InSPIRE].

[40] S. Stieberger and T.R. Taylor, Closed String Amplitudes as Single-Valued Open String Amplitudes, Nucl. Phys. B 881 (2014) 269 [arXiv:1401.1218] [INSPIRE].

[41] F. Brown and C. Dupont, Single-valued integration and superstring amplitudes in genus zero, arXiv: 1810.07682 [INSPIRE].

[42] J. Broedel, O. Schlotterer, S. Stieberger and T. Terasoma, All order $\alpha^{\prime}$-expansion of superstring trees from the Drinfeld associator, Phys. Rev. D 89 (2014) 066014 [arXiv: 1304.7304] [INSPIRE]. 